\title{
Estudio arqueológico no invasivo mediante la reconstrucción virtual tridimensional de ocho urnas cinerarias prehispánicas de la Tierra Caliente michoacana, México
}

Non-invasive Archaeological Study through Tridimensional Virtual Reconstruction of Eight Pre-Columbian Funerary Urns from Tierra Caliente, Michoacan, Mexico

José Luis Punzo Díaz

Centro INAH-Michoacán,

Instituto Nacional de Antropología e Historia (INAH), México

jose_punzo@inah.gob.mx

\section{Alfonso Gastélum-Strozzi}

Centro de Ciencias Aplicadas y Desarrollo Tecnológico (Ccadet),

Universidad Nacional Autónoma de México (UNAM), México

alfonso.gastelum@ccadet.unam.mx

Ingris Peláez Ballestas

Servicio de Reumatología,

Hospital General de México "Dr. Eduardo Liceaga" (HGMEL),

Secretaría de Salud, México

ingris.pelaez@salud.gob.mx

Jesús Zarco Navarro

Centro INAH-Michoacán,

Instituto Nacional de Antropología e Historia (INAH), México

jesuszarconavarro@hotmail.com

\section{Resumen}

Esta INVESTIGACIÓN presenta los resultados de la aplicación de técnicas de imagenología y el desarroIlo de métodos computacionales para el análisis de los depósitos arqueológicos contenidos en ocho urnas cinerarias prehispánicas descubiertas en el municipio de Huetamo, Michoacán, México. Gracias a este estudio se generaron, previo a su microexcavación, modelos digitales tridimensionales precisos de los contenidos de los contextos arqueológicos: un total de 5426 entidades, entre las que destacan restos óseos, artefactos de metal y otros que formaban parte de las ofrendas funerarias. Así, esta nueva técnica de documentación tridimensional no invasiva se perfila dentro del campo de la 
arqueología no sólo como una importante fuente para la investigación, sino también como una relevante herramienta de conservación.

\section{Palabras clave}

arqueología de Michoacán; arqueología no invasiva; bioarqueología; cremaciones; modelado 3D; conservación arqueológica

\section{Abstract}

This RESEARCH presents the results of the application of imaging techniques and the development of computational methods for the analysis of archaeological deposits contained within eight Pre-Columbian cinerary urns discovered in the municipality of Huetamo, Michoacan, Mexico. From this study, precise tridimensional digital models of the contents of the archaeological contexts were generated prior to their micro-excavation. These contents included a total of 5426 entities, among which bone remains, metal artefacts, and others that formed part of the funerary offerings, stand out. Thus, this new non-invasive tridimensional documentation technique is set to be not only an important source for the investigation, but also a relevant conservation tool.

\section{Key words}

archaeology; Michoacan; non-invasive archaeology; bioarchaeology; cremations; 3D modeling; archaeological conservation

\section{Introducción}

E n los últimos años tanto el uso de nuevas tecnologías como la introducción de perspectivas transdisciplinarias en la arqueología han impulsado grandes avances en la investigación y la conservación de los objetos recuperados por medio de excavaciones (Reindel y Wagner 2009; Grosman 2016; Errickson y Thompson 2017). Esta perspectiva permea la presente INVESTIGACIÓN, que resulta de la interacción de un grupo de investigadores de distintas disciplinas (arqueología, ciencias de la computación, física, medicina, antropología física, restauración) que en últimas fechas hemos estado en busca de caminos novedosos con que obtener un máximo de información arqueológica con el menor detrimento posible de los artefactos.

La utilización de las técnicas de imagenología en arqueología está documentado desde la década de 1960: primero, la aplicación de rayos $\mathrm{X}$ al estudio de restos óseos, posteriormente, la tomografía axial computarizada (TAC) y, más recientemente, la resonancia magnética (RM): los estudios arqueológicos que hacen uso de estas técnicas han ido en aumento, de la misma manera en que ha mejorado la calidad de los resultados (Errickson y Thompson 2017). La reconstrucción 3D se ha utilizado a partir de los datos generados por TAC o RM en los estudios de superficies de excavación, el arte rupestre y la descripción de artefactos cerámicos, óseos y líticos (Reindel y Wagner 2009; Grosman 2016). Además, los al- goritmos diseñados para la reconstrucción en 2D y 3D han permitido la especificación cuantitativa de los materiales arqueológicos y el desarrollo de programas de uso fácil para el estudio más detallado de los artefactos (por ejemplo, análisis geomorfométricos) y el diseño de tipologías sin alterar las piezas originales (Grosman et al. 2008, 2014). No obstante que tanto la TAC como la RM son técnicas útiles, tienen limitantes: por ejemplo, el uso de esta última en contextos que incluyen objetos de metal, aparte de su alto costo comparado con la TAC y su disponibilidad comercial.

La TAC se ha aplicado mundialmente en el estudio de contextos funerarios, por ejemplo, cementerios, fardos funerarios y restos óseos individuales (Errickson y Thompson 2017); en México, para algunos estudios de fardos funerarios del centro del país (Peñaloza Meza 2015) y urnas cinerarias de gran tamaño en el norte (Villalpando 2016; Martínez y Hernández 2016). Es pertinente aclarar que los estudios realizados en México y en contextos funerarios de pequeñas dimensiones en el mundo no han previsto el desarrollo de algoritmos que permitan la reconstrucción 3D de los elementos al interior de los mismos. El uso de la TAC también se ha practicado con momias y fardos funerarios para la elaboración de grandes bases de datos y la realización de estudios morfométricos y de paleopatología (Grosman 2016).

Para el presente estudio se tomó como elemento de análisis un conjunto de vasijas que contienen los restos cremados de personas que habitaron hace cerca de 1000 años en las cercanías del río Balsas, hallados durante la excavación arqueológica del sitio de Los Tamarindos en el año 2015. Aquéllas se analizaron mediante técnicas no invasivas que permitieron documentar tridimensionalmente los datos contextuales, amén de su preservación, equilibro relevante si se considera que el proceso de excavación en arqueología implica por sí mismo una destrucción del contexto en el cual se encuentran los artefactos (Punzo Díaz et al. 2016). Cabe destacar la importancia arqueológica de la zona del río Balsas medio, donde se ha encontrado una de las mayores densidades de sitios en la región, con una alta diversidad en cuanto a su tamaño y tipo, cuya historia, desafortunadamente, se desconoce casi por completo (Punzo Díaz et al. 2016); en este sentido, el presente estudio pretende aportar de manera puntual al conocimiento de una tradición funeraria a través de la cremación, de la cual prácticamente no se contaba con dato alguno hasta el momento.

Aquí buscamos presentar una técnica novedosa de estudio mediante imagenología compleja para la reconstrucción de objetos en 3D a través de TAC. En este sentido, se presentará de forma general el contexto de los hallazgos de las urnas funerarias, así como la complejidad de su estudio por métodos tradicionales, para exponer la metodología de la construcción de los modelos 3D y el análisis de estas piezas, dando cuenta de las enormes 


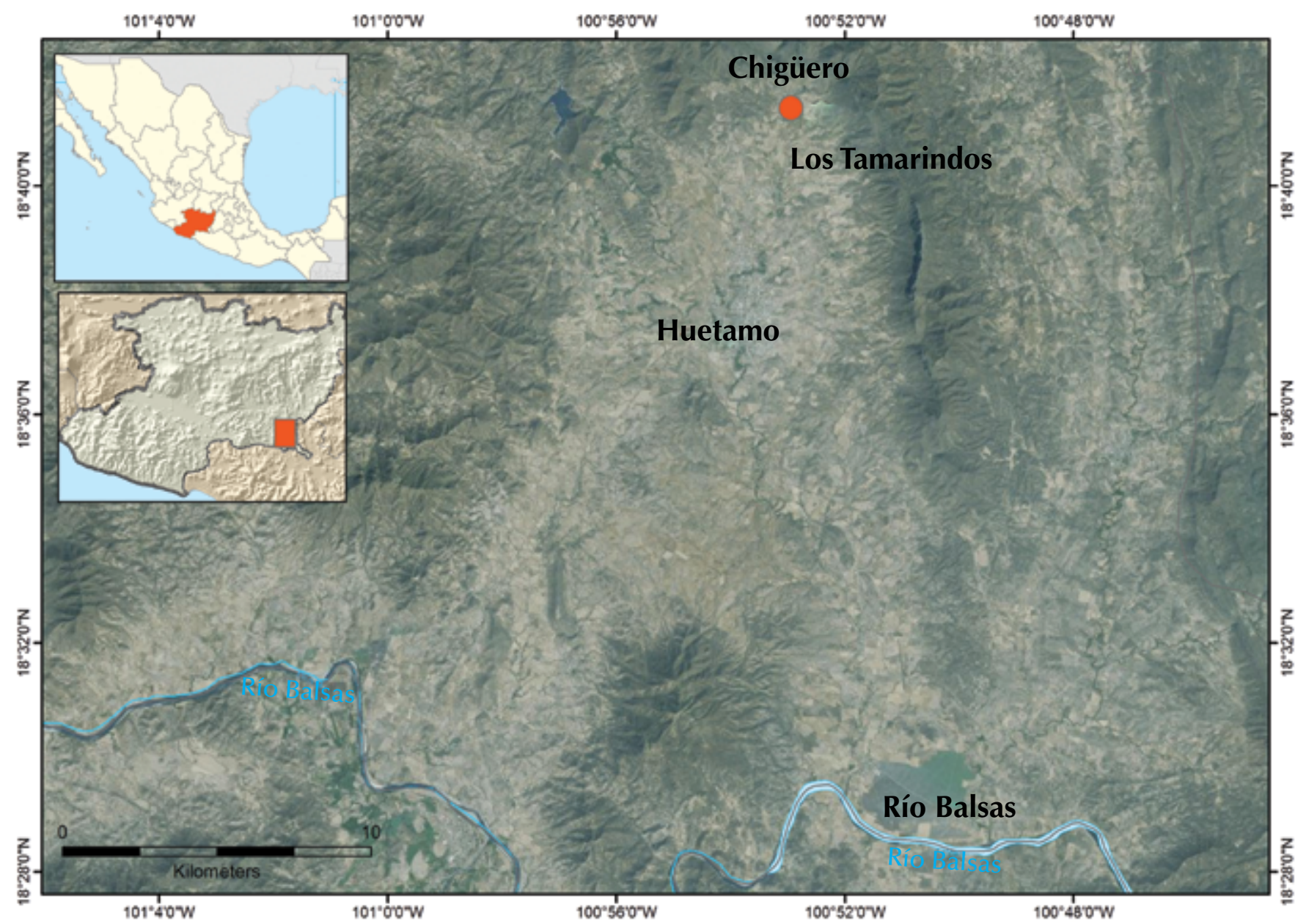

FIGURA 1. Mapa de ubicación de la zona de estudio (Mapa: José Luis Punzo, 2016; cortesía: Centro INAH-Michoacán, México).

ventajas y el potencial de esta técnica para los campos de la arqueología y la conservación.

\section{Antecedentes: el proyecto de salvamento arqueológico Presa del Chigüero, Huetamo, Michoacán, México}

Desde la constitución de la Comisión de Desarrollo de la Cuenca del Río Balsas en los años sesenta del siglo pasado, cuando fue su vocal Lázaro Cárdenas del Río, se planteó la necesidad de construir una presa de riego al norte de la población de Huetamo, sobre el arroyo del Chigüero, la que se canceló en distintas ocasiones. ${ }^{1}$ No fue sino hasta principios del actual sexenio (2012-2018) cuando se reactivó el plan, con la autorización a la Comisión $\mathrm{Na}$ cional del Agua (Conagua). Así, concomitantemente, en el 2014 se iniciaron las gestiones con el Instituto Nacional de Antropología e Historia (INAH), México, para ejecutar un proyecto de salvamento en la zona de afectación (Punzo Díaz et al. 2015; Punzo Díaz et al. 2016).

1 Esto de acuerdo con lo expresado por los habitantes de la comunidad del Chigüero.
Durante los años 2015 y 2016, un equipo de arqueólogos del Centro INAH-Michoacán, dirigido por el arqueólogo José Luis Punzo, Ilevó a cabo dichos trabajos de salvamento en el que luego sería el emplazamiento de la presa de riego del Chigüero (Figura 1). La región del río Balsas medio es una de las registradas en el estado de Michoacán como de muy alta densidad de vestigios arqueológicos, por lo que, como era de esperarse, durante los recorridos realizados en la zona de afectación por la construcción de la cortina de la presa, los diques, el área de inundación, así como el distrito de riego, se localizaron 59 sitios arqueológicos en superficie. Por las alteraciones que habrían de sufrir, se determinó realizar excavaciones en 10 de ellos, cuyos resultados fueron muy amplios y ya se han reportado en otras publicaciones e informes (Punzo Díaz et al. 2015, 2016).

Como parte de dichos trabajos, precisamente donde se determinó el área de construcción del dique número 2 de la presa, se localizó un pequeño sitio arqueológico, denominado Los Tamarindos, el cual, aunque apenas contaba con perceptibles vestigios arqueológicos en superficie, fue objeto de trabajos de excavación. 
Excavaciones arqueológicas en el sitio de Los Tamarindos, Huetamo, Michoacán

Justamente en la parte alta y plana de una loma al costado este del arroyo del Chigüero, municipio de Huetamo, Michoacán, se localiza el sitio arqueológico de Los Tamarindos, hallado en el 2015 durante un recorrido de superficie en el que se observaron dos pequeños montículos, ubicados al este y oeste respectivamente, ambos de forma circular en planta y construidos con piedra y tierra, que parecían ligeras plataformas similares a otras de la región (cfr. Punzo Díaz et al. 2016). Desafortunadamente, los montículos de Los Tamarindos estaban muy deteriorados, pues en la década de los noventa su superficie se aplanó y limpió para dar lugar a una cancha de juego de pelota tarasca, abandonada años después: en la actualidad este espacio está dedicado a actividades agrícolas (cfr. Punzo Díaz et al. 2016).

En consecuencia, se propuso conformar dos unidades de excavación, una para cada montículo, con el fin de determinar su sistema constructivo y, si fuera dable, establecer el tipo de uso que tuvieron. Respecto de la primera, en el montículo oeste se realizó una cala de aproximación en uno de sus costados, la cual no alcanzó gran profundidad debido a que la roca madre se encontraba ya a 30-40 $\mathrm{cm}$ de profundidad. Sin embargo, gracias a la excavación se notó que los antiguos pobladores de esta región colocaban como elementos constructivos, a manera de cimiento, tanto piedras de diversos tamaños como fragmentos de instrumentos: manos de metate, aguzadores y discos de roca caliza cementados y cubiertos por tierra, para incrementar el volumen del montículo (cfr. Punzo Díaz et al. 2016).

La segunda unidad se situó en la parte más alta del montículo este. Durante la excavación de los primeros estratos se descubrieron materiales arqueológicos tanto prehispánicos como modernos; sin embargo, en el más profundo (aproximadamente a $40 \mathrm{~cm}$ ) se halló un contexto prehispánico, conformado por un cajete y una olla de cerámica, ambos monocromo café (cfr. Punzo Díaz et al. 2016). Estos objetos estaban colocados directamente sobre el tepetate: el cajete, bocabajo, a manera de tapa, cubriendo un conjunto de huesos humanos cremados, sin ceniza, que se habían depositado en una cavidad hecha intencionalmente sobre el tepetate (Figura 2); la olla —que se halló a unos centímetros al suroeste del cajete durante el proceso de descubrimiento del tepetate- estaba colocada de manera intencional para cubrir una cavidad. Debido a la presencia de fisuras, se observó que en el interior de la olla se hallaban restos de huesos y cenizas (cfr. Punzo Díaz et al. 2016).

Por lo anterior, se tomó la decisión de realizar una excavación extensiva, en un área de $92 \mathrm{~m}^{2}$, en la que se encontraron dos estratos claramente diferenciados. En el primero se hallaron elementos arquitectónicos que indicaban la presencia de un muro de piedra, así como ma-

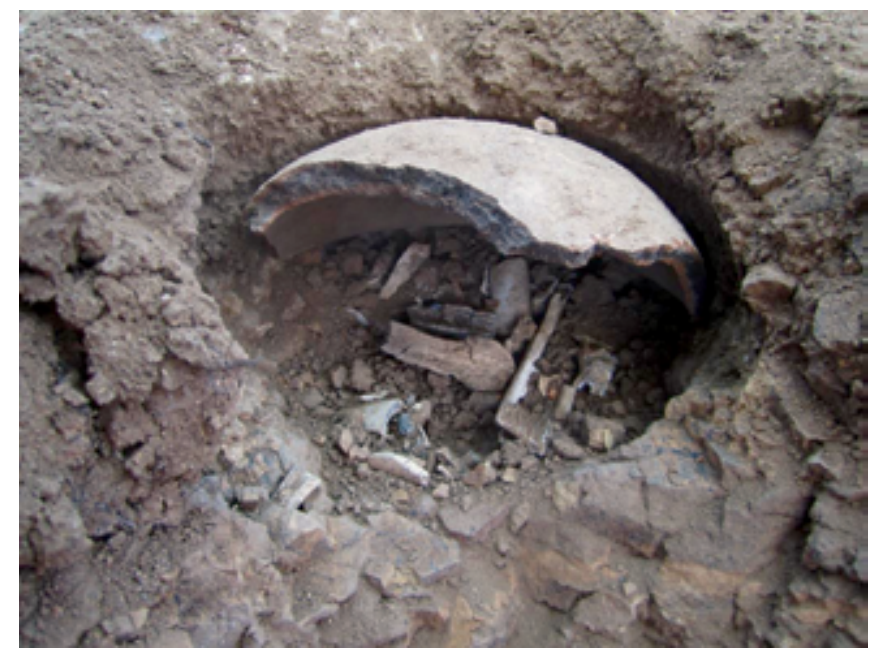

FIGURA 2. Vasija cubriendo restos óseos cremados, depositados directamente en un hoyo en la roca madre, Los Tamarindos (Fotografía: Jesús Zarco, 2015; cortesía: Centro INAH-Michoacán, México).

teriales arqueológicos diversos, tales como restos de cerámicas, monocromas en su mayoría, aunque también algunos restos de cerámicas decoradas que pudimos relacionar con tipos usados durante el periodo Posclásico (tipos Copujo y Yarahuato), lascas de percusión extraídas de nódulos de andesita y pedernal, láminas de percusión, fragmentos de núcleos prismáticos y navajillas prismáticas de obsidiana (cfr. Punzo Díaz et al. 2016).

El segundo estrato, que se excavó hasta el contacto con la roca madre y que en su parte más profunda llegó entre los 60 y $40 \mathrm{~cm}$ respecto de la superficie, se hizo el hallazgo de un total de 42 urnas cinerarias en un espacio de apenas $30 \mathrm{~m}^{2}$ (Figura 3).

Las urnas estaban colocadas sobre la roca madre en pequeñas horadaciones que les servían de base; la gran mayoría corresponde a ollas de distintas formas, sin decoración, cubiertas con un cajete a manera de tapa y tal vez de cuchara para introducir los restos. Asimismo, de forma aislada se encontraron una figurilla antropomorfa de cerámica, tres dientes humanos, dos aros y tres cascabeles de cobre, uno de los cuales mostraba evidencia de haber estado cubierto con un textil.

La distribución de las urnas permite plantear al menos dos posibilidades: la primera, que se dispusieron en un solo acto de deposición, puesto que siguen un patrón norte-sur, con una separación de pocos centímetros entre unas y otras (cfr. Punzo Díaz et al. 2016), o, segunda, que se trate de un cementerio de cremaciones con una organización muy estructurada, al grado de que permitió a los antiguos habitantes de la zona llevar a cabo una deposición controlada a lo largo de un periodo de tiempo más amplio. No podremos asegurar estas hipótesis hasta tener fechamientos absolutos de un vasto número de ellas, documentación que se realizará en una etapa posterior del estudio.

De esta manera los trabajos de excavación extensiva en la unidad 2 (montículo este) determinaron la existencia 


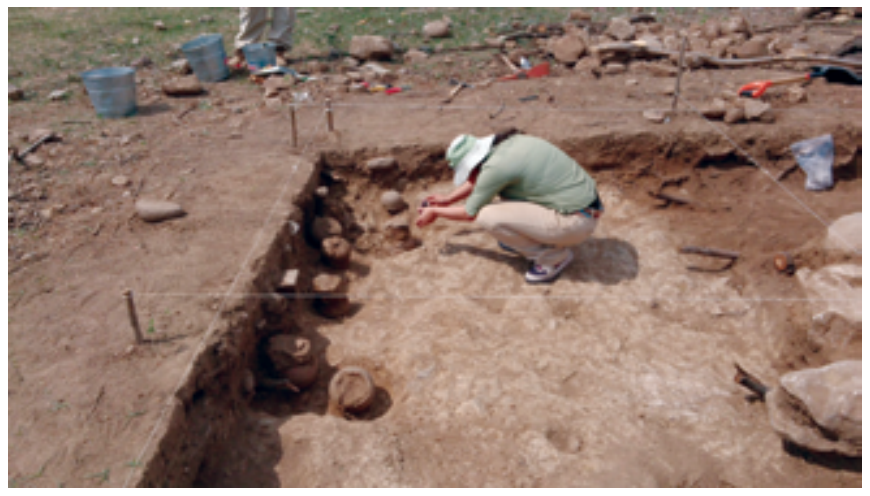

FIGURA 3. Distribución de vasijas en la unidad 2 de la excavación Los Tamarindos (Fotografía: Jesús Zarco, 2015; cortesía: Centro INAHMichoacán, México).

de un área de enterramiento. Es importante recalcar que, en tanto que no pudimos localizar hasta el momento los sitios de las piras crematorias u otros elementos ligados al tratamiento funerario - cuyos antecedentes se describirán a continuación-, se trata de un acto final del proceso de cremación que se debió de practicar en la zona.

\section{Las cremaciones funerarias en el Michoacán prehispánico}

La cremación funeraria para deposición en urnas fue una práctica que existió en México desde hace más de 3000 años (Duncan et al. 2008:5315). En el Michoacán prehispánico destacan los hallazgos hechos en la ciénaga de Zacapu, en el sitio de Loma Alta, de un gran número de urnas cinerarias que, al igual que nuestro caso de estudio, contenían huesos triturados y cenizas (Arnauld et al. 1993:87-148). Sin embargo, las urnas de Loma Alta poseen una temporalidad que las remonta a los primeros 200 años de nuestra era, son de gran tamaño y, en varios casos, contuvieron más de un individuo (Carot y Susini 1989:115).

Al parecer, al final del periodo Clásico (100 a. C.-500 d. C.) y en el Epiclásico (500-900 d. C.) se presentó una discontinuidad muy marcada en el uso de las cremaciones, ya que predominó la disposición de entierros múltiples en grandes tumbas, como los reportados en el sitio de Guadalupe (Pereira 1997) o en Tingambato (Piña Chan y Oi 1982), entre otros.

Para la Tierra Caliente de los estados de Michoacán y Guerrero, Brand (1942) menciona la presencia de restos humanos cremados y depositados en urnas cerámicas en la región del Balsas (cfr. Bonfil Olvera 2004:35): aquí la cremación parece ser un fenómeno tardío, lo cual se fundamenta en que no se observaron rastros de esta práctica de enterramiento en las excavaciones de los sitios arqueológicos de La Yácata de la Casita, el Ancón o Loma de Piritícuaro, fechados entre el 200 y el 900 d. C. (Punzo Díaz en prensa).

En relación con el periodo tardío en Michoacán, es decir, al momento de la llegada de los españoles, tene- mos evidencia muy esporádica sobre la práctica de cremaciones. Aunque la Relación de Michoacán la describe como la práctica funeraria más importante, ya que correspondía al cazonci de Tzintzuntzan (Alcalá 2008); seguramente se realizaba de forma reservada para una parte muy limitada de la población (Pereira 2017 en prensa), ya que no es común encontrarla en los entierros de otro tipo de segmentos de la población. Un ejemplo singular es la gran concentración de huesos quemados y rotos que se encontraron en el relleno de la esquina noroeste de la Gran Plataforma de Tzintzuntzan, los cuales debieron haber formado parte de los grandes rituales que se hicieron en la capital del señorío tarasco hacia el final de la época prehispánica (Acosta 1939; Castro-Leal 1986).

Ahora bien, tomando en cuenta la información precedente, la datación preliminar de las cremaciones del sitio Los Tamarindos se ha estimado en un rango con límites aproximados. Con base en el contenido de los objetos metálicos observados en este contexto funerario, la fecha más antigua posible se ubica hacia el año $900 \mathrm{~d}$. C., la más temprana que podemos suponer para la región (Hosler 1994:170). Cabe señalar que durante el Posclásico Tardío el señorío tarasco le otorgó a grupos matlatzincas del Valle de Toluca, a cambio de rendir servicio militar en sus ejércitos tarascos, diversas regiones de la Tierra Caliente (Quezada 1996:44). Adicionalmente, respecto de las cremaciones funerarias, sabemos que los grupos tarascos y matlatzincas "practicaban este método esporádicamente" (García Payón 1941:64); por lo que podría tratarse de vasijas pertenecientes a las tradiciones seguidas por alguno de estos grupos. Por otro lado, la tipología de los cascabeles recuperados - de tipo globular- se asocia con el periodo previo al año $1300 \mathrm{~d}$. C. Así, se estima que la datación relativa de nuestro caso de estudio corresponde, inicialmente, al Posclásico (cfr. Punzo Díaz et al. 2016).

\section{La microexcavación en las urnas funerarias de Los Tamarindos y su problemática}

Una vez que se llevó a cabo el rescate arqueológico de las 42 urnas, con su contenido intacto, en el laboratorio del Centro INAH-Michoacán se inició su estudio por métodos tradicionales de microexcavación (McKinley 1993, 1997). El proceso se llevó a cabo inicialmente en 8 urnas, con el fin de reconocer los patrones funerarios en la región, dejando en ese momento las 34 restantes para futuras excavaciones.

La microexcavación es un proceso detallado de desenterramiento en un espacio reducido, realizado generalmente en condiciones controladas de laboratorio, durante el cual se hace un registro minucioso de los restos óseos, los contenidos de las vasijas, las muestras de tierra y demás elementos relacionados con el objeto de estudio (Barker 1977; Joukowsky 1980; McKinley 1993, 1997, 2013). En nuestro caso, se realizó con el fin tanto de recuperar de forma controlada y precisa restos óseos, ma- 
teriales arqueológicos que pudieran formar parte de la ofrenda y muestras diversas de sedimentos (incluidos polen y fitolitos) para datación absoluta por radiocarbono, análisis paleoetnobotánico (flotación y observación al microscopio óptico), material genético (ADN) e isótopos estables, como con la intención de establecer con mayor precisión el fechamiento del suceso funerario, así como la edad, el sexo, las posibles patologías, las dietas y los hábitos de los individuos enterrados. Por ello, dicho proceso requirió un método estratigráfico muy fino, así como medidas estrictas de asepsia para evitar la contaminación de muestras. Sin embargo, las pequeñas dimensiones de las urnas y el difícil acceso, por lo cerrado de la boca de éstas, dificultaba mucho este trabajo.

Estos parámetros se siguieron en la microexcavación de las ocho urnas mencionadas (Figura 4), lo que dio elementos para empezar a entender el patrón de deposición de los huesos y la ceniza. En este sentido se observó un alto grado de trituración de los huesos, así como su forma de deposición en el fondo de las vasijas, mezclados con una muy baja cantidad de ceniza. A la par, se recuperaron distintos artefactos que se colocaron a manera de ofrendas, tales como cascabeles y aros de cobre, una placa de piedra tallada, figurillas, un sartal de seis conchas de la especie Anadara brasiliana, procedente del océano Atlántico. Asimismo, se recogieron pequeños fragmentos de carbón y restos de tierra batida - posiblemente, fragmentos de bajareque o adobes, que son materiales constructivos documentados en el resto de nuestras excavaciones- (Punzo Díaz et al. 2015), elementos muy relevantes por razón de que, en tanto pueden considerarse como restos de las piras crematorias, brindan información acerca de la transformación en el proceso de cremación (McKinley 2013:4-5).

Sobre los huesos rescatados en las distintas urnas, fue posible notar coloraciones desiguales adquiridas por el propio proceso de cremación, ya sea por oxidación o calcinación: hueso blanco, hueso azul y hueso negro

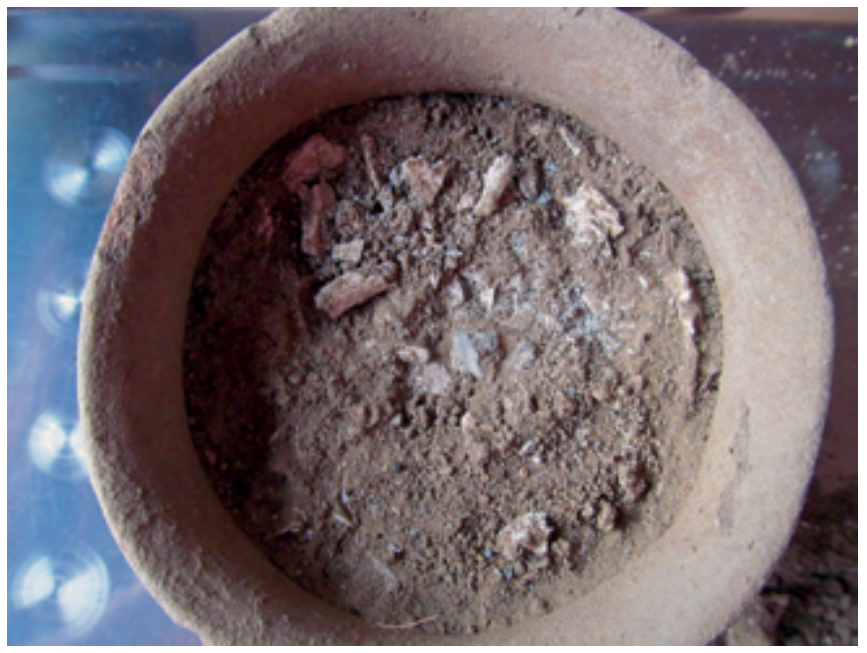

FIGURA 4. Proceso de microexcavación de una urna cineraria (Fotografía: José Luis Punzo, 2015; cortesía: Centro INAH-Michoacán, México). o blanco con negro (McKinley 2013:3). La mayor de estas piezas mide aproximadamente $4 \mathrm{~cm}$ de largo, lo que indica que el material óseo se trituró, después de la cremación, y se mezcló con la ceniza para introducirlo en las urnas, en las que posteriormente se dispusieron las ofrendas. En relación con el estudio de antropología física de los huesos podemos señalar, por el momento, que los que provienen de las urnas 16 y 28 son de infantes. De esta última urna se envió una muestra de hueso calcinado para fechamiento por radiocarbono, que dio un resultado de $609 \pm 26$ AP, o calibrado con dos sigmas y una certeza del 95\% 1296-1404 d. C. $(2 \sigma),{ }^{2}$ lo que, junto con los resultados del análisis cerámico y los objetos de metal mencionados, confirma que las urnas debieron haberse depositado durante el periodo Posclásico Medio, poco antes de la expansión tarasca en esta región de la Tierra Caliente.

Ahora bien, debe señalarse que durante la microexcavación, aunque se realizó minuciosamente, fue muy difícil mantener un control microestratigráfico tan riguroso que, a su vez, permitiese el registro arqueológico de datos con la precisión requerida para entender el proceso de deposición de los huesos, las cenizas y, especialmente, de las ofrendas. Ello se debió a diversas contrariedades: el tamaño muy reducido de las vasijas, particularmente de sus bocas, no permitía un campo de excavación adecuado; la naturaleza del depósito arqueológico — mezcla de tierra, ceniza y hueso- propició la consolidación de la matriz, que contrastaba con la pérdida de cohesión de los huesos: la diferencia de dureza hacía muy complicada la excavación y el rescate de elementos.

Debido a ello, con la idea de optimizar un análisis deposicional más controlado se planteó la idoneidad de buscar técnicas imagenológicas con que describir el contexto al interior de cada urna cineraria, antes de proceder a la microexcavación. Esta segunda fase del proyecto, que incluyó la colaboración interinstitucional, se describe a continuación.

\section{Estudio arqueológico no invasivo mediante tomografía axial computarizada (TAC)}

Como primera estrategia de esta segunda fase de la investigación se valoraron varias técnicas imagenológicas, entre ellas, el uso de rayos X, TAC y RM, con el apoyo de la doctora Ingris Peláez Ballestas (Hospital General de México); después de considerar el beneficio en cuanto a la calidad de información, así como los costos y la factibilidad, se tomó la decisión de utilizar la técnica TAC en las ocho urnas cinerarias, de las 34 que se habían

\footnotetext{
${ }^{2}$ Los huesos que han sido calentados a más de $600^{\circ} \mathrm{C}$ por tiempo suficiente queman el colágeno, grasas y proteínas. La osteocalcita (apatita) en el hueso se convierte en carbonato estructural, el cual es fechado. Este método ha sido publicado y aceptado como de gran confiabilidad en 2000 en la 17th International Radiocarbon Conference (cfr. Beta Analytic s.f.).
} 
dejado en reserva. Esta técnica de imagenología ha sido utilizada para el estudio de urnas funerarias por diferentes investigadores en distintas partes del mundo, y se la considera una de las más apropiadas para esta clase de contextos (por ejemplo: McKinley 2013; Cerezo-Román y WiIliams 2014; Errickson y Thompson 2017). A partir del análisis preliminar de las imágenes obtenidas por la TAC, se observó la complejidad que presentaban estos contextos y se determinaron ciertos patrones ${ }^{3}$ de distribución de los huesos cremados y triturados, así como de la deposición de ceniza y las ofrendas. No obstante, algunos detalles aparecían difusos, lo que exigía explorar mediante tecnologías de imagenología más complejas.

Fue entonces cuando se inició una colaboración académica con los investigadores Alfonso Gastélum Strozzi, Miguel A. Padilla Castañeda y Jorge Alberto Márquez Flores, del Centro de Ciencias Aplicadas y Desarrollo Tecnológico (Ccadet-UNAM), México, cuyo objeto fue desarrollar métodos computacionales que permitieran el análisis de las imágenes obtenidas de la adquisición tomográfica (Figura 5).

Este proceso significó un desarrollo matemático, a saber:

Mediante la utilización de las imágenes adquiridas del sistema TAC se estableció una serie de volúmenes $\mathbf{V}_{\boldsymbol{u}} \in \mathbb{Z}^{3} \boldsymbol{u}=\left\{1, \ldots, N_{\boldsymbol{u}}\right\}$, donde $N_{\boldsymbol{u}}$ es el número de urnas adquiridas. Cada volumen $\mathbf{V}_{\boldsymbol{u}}$ está compuesto por un conjunto de voxeles $\Upsilon_{\boldsymbol{u}} \in \mathbf{V}_{\boldsymbol{u}}$, con $\boldsymbol{i}=\left\{1, \ldots, M_{\boldsymbol{u}}\right\}$, donde $M_{\boldsymbol{u}}$ es el número de voxeles por urna. A su vez, cada voxel $\Upsilon_{u i}$ está determinado por una tupleta dada por $\left(\left(X_{\boldsymbol{u} i}, \Upsilon_{\boldsymbol{u} \boldsymbol{i}}, Z_{\boldsymbol{u} \boldsymbol{i}}\right), V_{\boldsymbol{u} \boldsymbol{i}}\right)$, donde $\left(X_{\boldsymbol{u} \boldsymbol{i}}\right.$, $\left.\Upsilon_{u i}, Z_{u i}\right)$ da la posición del voxel $\Upsilon_{\boldsymbol{u}}$ en el volumen de la urna $\boldsymbol{u}, \mathrm{y}$ $\mathrm{V}_{\boldsymbol{u} \boldsymbol{i}}=\Omega\left(X_{\boldsymbol{u} \boldsymbol{i}}, \Upsilon_{\boldsymbol{u} \boldsymbol{i}}, Z_{\boldsymbol{u} \boldsymbol{i}}\right)$ es un atributo

${ }^{3}$ En este sentido, pudimos percatarnos de la poca homogeneidad en los contenidos de las urnas, como la mayoría de los huesos mayores fueron colocados en la parte inferior de las urnas y las ofrendas metálicas en la mayoría de los casos fueron colocados en la parte superior.

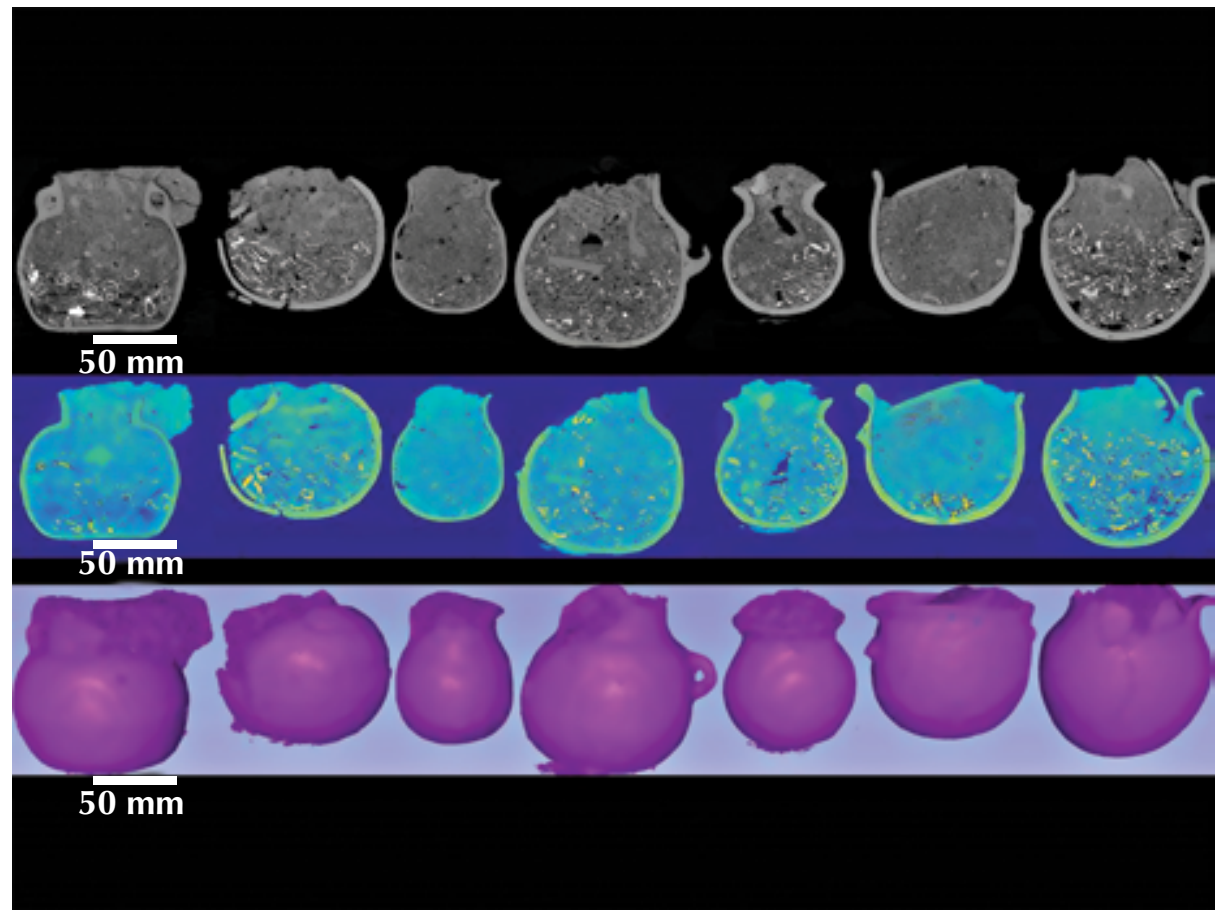

FIGURA 5. Estudio mediante escáner tomográfico computarizado y reconstrucción en 3D de las urnas (Imagen: Alfonso Gastélum, 2016; cortesía: Centro de Ciencias Aplicadas y Desarrollo Tecnológico [Ccadet-unam], México).

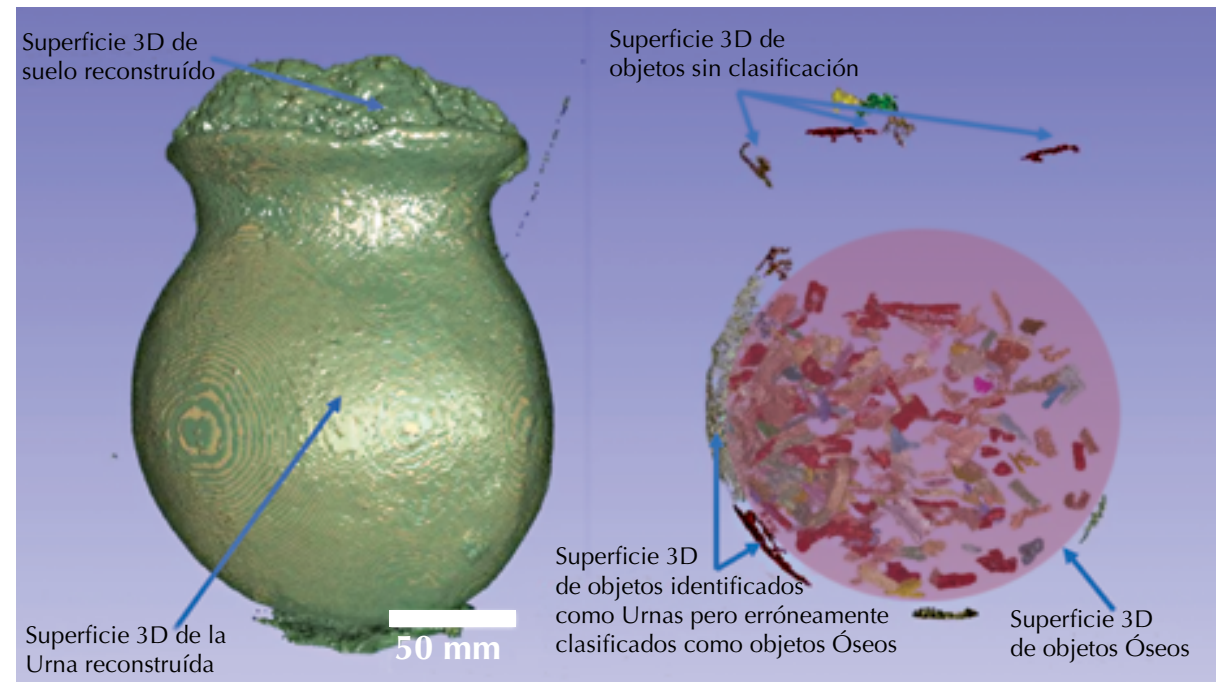

FIGURA 6. Reconstrucción en 3D de urna cineraria, imagen exterior e interior (Imagen: Alfonso Gastélum, 2016; cortesía: Centro de Ciencias Aplicadas y Desarrollo Tecnológico [Ccadet-UNAM], México).

escalar, o una etiqueta, que en el caso de las imágenes obtenidas por TAC $\mathrm{V}_{\boldsymbol{u} \boldsymbol{i}}$ define un valor escalar de intensidad, relacionado, a su vez, con la densidad de los objetos representados por éste. Las diferentes combinaciones de densidad presentes en el volumen original de cada urna se representan por voxeles, con un intervalo dinámico de 16 bits (Figura 6).

La finalidad en este punto era lograr por medio de un algoritmo el etiquetado de los objetos que conforman la urnas. Existen diversos métodos para ello, así como para la clasificación de datos, entre ellos, la binarización simple, cuando se cuenta con el problema de clasificar la imagen en dos clases, el ob- 
jeto de interés y el fondo; entre estos métodos podemos encontrar la implementación de Otsu (1979), la clasificación por watersheds aplicada al trabajar en objetos que se sobreponen unos a otros (Beucher 1992), y las clasificaciones, ya por medio de patrones de textura (Malik et al. 2001), ya de datos en grupos similares por medio de propiedades texturales (Dunn 1973).

La información de las urnas está formada por múltiples objetos sin un patrón textural específico, así que se decidió desarrollar un algoritmo basado en la clasificación de texturas por métodos de agrupación utilizando un clasificador difuso fuzzy - c - means (Dunn 1973) para etiquetar y clasificar los diferentes voxeles de acuerdo con sus valores de intensidad: éstos se emplean como el parámetro de clasificación debido a la relación existente entre la densidad física del objeto definido por el voxel y la intensidad de éste; los voxeles que conforman los objetos dentro de cada urna se clasifican en los siguientes tipos de objetos:

$$
L\left(\Upsilon_{u i}\right)=\left\{\begin{array}{l}
L\left(\Upsilon_{u i}\right)=0: \text { Clasificado como aire vacio } \\
L\left(\Upsilon_{u i}\right)=1: \text { Clasificado como tierra/material orgánico } \\
L\left(\Upsilon_{u i}\right)=2: \text { Clasificado como cerámica } \\
L\left(\Upsilon_{u i}\right)=3: \text { Clasificado como hueso } \\
L\left(\Upsilon_{u i}\right)=4: \text { Clasificado como metal }
\end{array}\right.
$$

Se usa un método difuso, ya que los voxeles discretos integran en ellos información proveniente de diversos componentes que conforman el volumen adquirido, lo que produce un efecto espacial de volumen parcial (Hoffman et al. 1979), donde la intensidad resultante en el voxel es la integración de densidades de diferentes materiales en diversas proporciones. Así, al clasificar los voxeles se considera que éstos pueden pertenecer a más de una clasificación, y el algoritmo de fuzzy - c - means (Dunn 1973) realizará iteraciones hasta que cada elemento (voxel) se asigne a su grupo y se defina un nuevo volumen con voxeles etiquetados.

El nuevo volumen $\boldsymbol{L}(\boldsymbol{u})$ de voxeles etiquetados se usó para la obtención de los diferentes objetos definidos por voxeles con valor igual a $L\left(\Upsilon_{u i}\right)=3$ o $L\left(\Upsilon_{u i}\right)=4$; cada objeto se definió por el conjunto de voxeles con la misma etiqueta con un nivel de conectividad de 16.

De cada urna se obtuvo una colección de objetos $O_{j}$ con $j=\left\{1, \ldots, H_{\boldsymbol{u}}\right\}$, donde $H_{\boldsymbol{u}}$ es el número de objetos por urna $\boldsymbol{u}$. Cada uno de ellos está formado por un subgrupo de voxeles, donde $\Upsilon_{u j i} \subset \Upsilon_{u i}$ : los voxeles que definen a un objeto se utilizan para la generación de superficies definidas por mallas triangulares tridimensionales $\Sigma_{j}$. Tales mallas se obtienen usando una implementación del método de marching cubes (Lorensen 1987; Lorensen y Cline 1987): la superficie $\Sigma_{j}$ está definida por la unión de triángulos $\boldsymbol{T}_{l}$, dado por $\Sigma_{j}:=U_{l} \boldsymbol{T}_{l}$, con $l=\left\{1, \ldots, N_{j}\right\}$ donde $N_{j}$ es el número de triángulos $\boldsymbol{T}_{l}$ que forman la superficie (Bærentzen et al. 2012).

Por lo tanto, los grupos de voxeles y la superficie 3D obtenidos por objeto sirvieron para medir, mediante la utilización de un algoritmo de medidas morfológicas desarrollado por Prado et al. (2016), las propiedades de forma (área de superficie, volumen del objeto, compacidad y esfericidad) de cada uno de éstos, y su posición y distribución dentro de cada uno de los volúmenes con los cuales es posible describir cuantitativamente el contexto.

Como resultado del tratamiento de las imágenes de TAC mediante estos tres algoritmos, se aisló, describió y ubicó imagenológicamente 5426 objetos al interior de las 8 urnas, lo cual incluyó la separación de los huesos de los materiales de ofrenda (Figura 7). La diagramación permitió, además de ubicar y estudiar el patrón de distribución dentro del conjunto tridimensional de la urna, hacer maquetas volumétricas de cada elemento: prototipos reales de los objetos que pueden materializarse en una impresora de tres dimensiones.

El sistema construido también hizo posible estudiar los componentes óseos en las urnas y obtener datos volumétricos de éstos: la Figura 8 muestra una colección de huesos en una zona de la urna número 2 , donde se presenta su propiedad de curvatura y son observables áreas de fracturas, mensurables digitalmente.

El método de estudio aplicado a las tomografías de las urnas toma un tiempo aproximado de dos a tres horas para pasar de las imágenes de tomógrafo a objetos tridimensionales con medidas morfológicas; la variación en el tiempo depende, por un lado, del volumen inicial que ocupa la urna y, por el otro, del número de objetos clasificados por el sistema para su medición.

Para la construcción aditiva de objetos tridimensionales, el tiempo que toma la obtención de un objeto en ácido poliláctico (PLA, por sus siglas en inglés) (Gupta et al. 2008) depende de la forma y el volumen de la pieza; por ejemplo, construir un cascabel toma de 1:30 a 2:00 horas, pero piezas pequeñas óseas o anillos pequeños pueden tomar desde 20 min hasta $45 \mathrm{~min}$. Los objetos se construyeron con una máquina modelo Ultimaker 1 (UItimaker 2017).

\section{A manera de conclusiones: posibilidades de investigación, divulgación y conservación}

La aplicación tecnológica que hemos desarrollado para el estudio arqueológico no invasivo de urnas funerarias del sitio arqueológico de Los Tamarindos ya permite ofrecer una serie de beneficios a seguir:

- Naturalmente, el primero y más importante es que es posible estudiar las características y la condición del contexto arqueológico sin necesidad de excavarlo y, por lo tanto, sin destruirlo.

- Describir con gran detalle todos los elementos depositados, creando un registro arqueológico detallado.

- Realizar estudios dimensionales y formales de cada elemento segmentado al interior de la urna, con lo que se discierne su volumen exacto en sus tres dimensiones. 


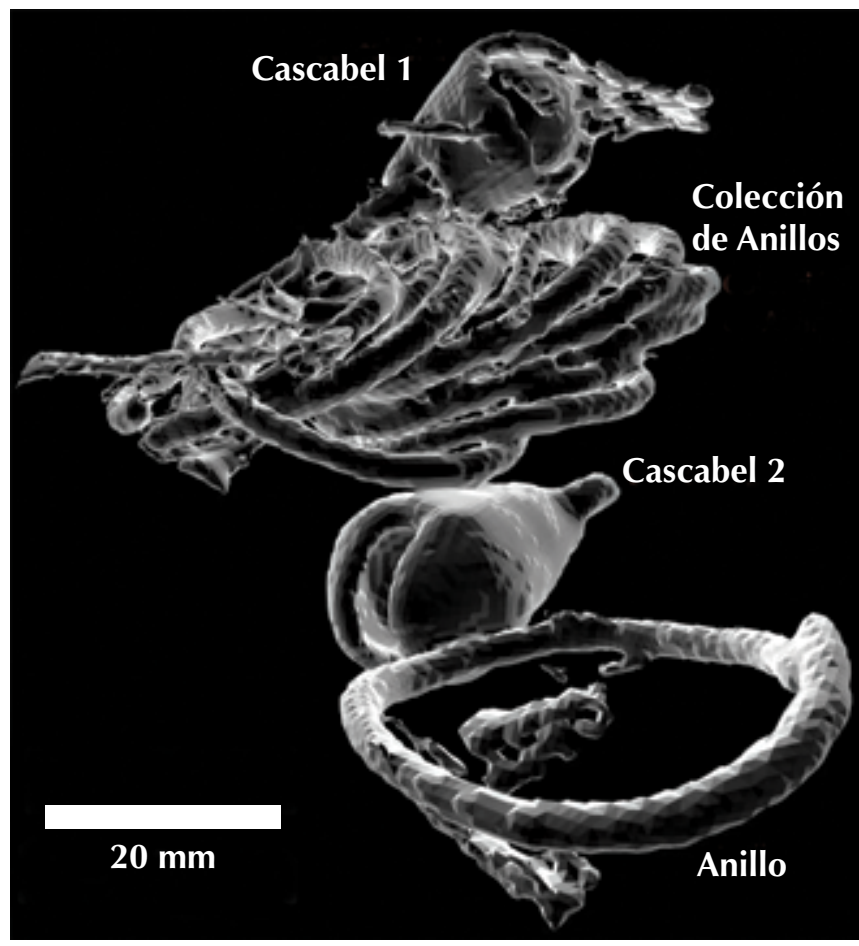

FIGURA 7. Reconstrucción de objetos metálicos al interior de una de las urnas (Imagen: Alfonso Gastélum, 2016; cortesía: Centro de Ciencias Aplicadas y Desarrollo Tecnológico [Ccadet-UnAM], México).
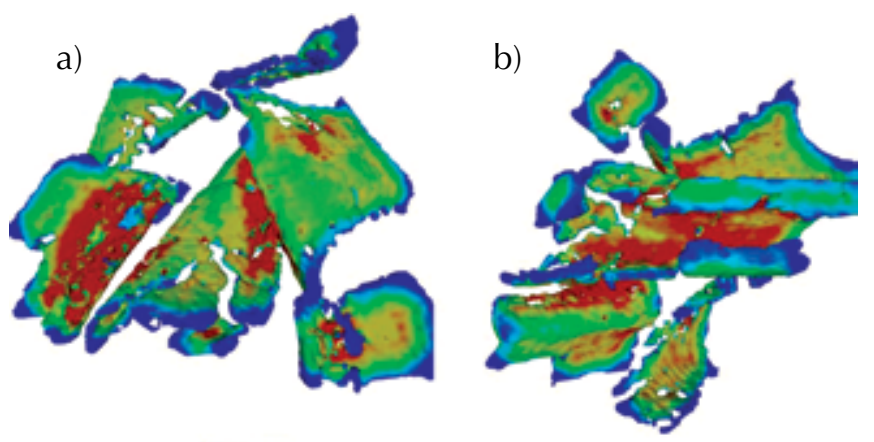

C)
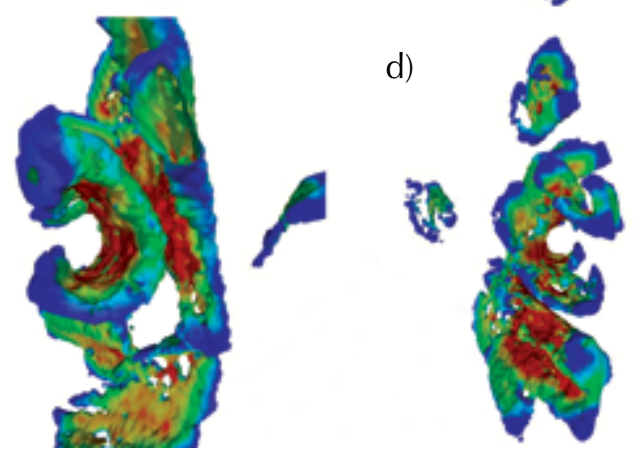

FIGURA 8. Los colores muestran el grado de curvatura normalizada del hueso; los valores rojos son los puntos de mayor curvatura local. En los huesos se pueden percibir fracturas y perforaciones. a) Vista frontal del hueso, b) vista posterior, c) vista lateral derecha, d) vista lateral izquierda (Imagen: Alfonso Gastélum, 2016; cortesía: Centro de Ciencias Aplicadas y Desarrollo Tecnológico [Ccadet-UNAM], México).
- La posibilidad de la impresión en tres dimensiones de los elementos segmentados permite no sólo reconstruir de forma física el mismo contexto, sino incluso llevar a cabo estudios de los objetos, tanto de los huesos como de las ofrendas ahí depositadas. En ese sentido, es posible hacer análisis tipológicos de los cascabeles y aros metálicos impresos.

- Gracias a la precisión del registro logrado a través de los algoritmos desarrollados se genera una imagen del contexto arqueológico en su condición actual: una reconstrucción que faculta el estudio no sólo dimensional y formal de las entidades contenidas, sino también de los espacios "vacíos" que quedan entre ellos, lo que proporciona datos interesantes para comprender los procesos tanto de deposición contextual funeraria como de su transformación a lo largo del tiempo.

- La segmentación de los elementos de forma individual y el estudio de forma contextual nos dan una enorme posibilidad de analizar este tipo de contextos complejos.

Tales beneficios no podrían haberse obtenido por medio de recursos imagenólogicos como rayos $X$ o RM. Los primeros sólo hubieran aportado la información de la distribución de los objetos al interior de las urnas, pero no realizar descripciones de cada objeto ni, menos aún, una reconstrucción en 3D. La RM, si bien fue una opción alternativa al uso de TAC, limitaron su uso, lamentablemente, el contenido de metales (en este caso, cobre) al interior de las urnas, y su alto costo y difícil disponibilidad. Cabe mencionar que el análisis de TAC y las técnicas computacionales requeridas siguen siendo costosas para la mayoría de los proyectos arqueológicos, lo que representa una desventaja importante, amén de que la colaboración con múltiples especialistas para el proceso e interpretación de los datos es una de sus condicionantes.

Actualmente el proceso de investigación mediante el uso de esta tecnología sigue en curso: se espera completar el análisis de las otras 26 urnas cinerarias que no se han excavado ni se les ha realizado el análisis de reconstrucción 3D; concluirlo nos otorgará una gran cantidad de información con la que complementar la que ya se tiene acerca de las culturas de la Tierra Caliente de Michoacán, así como el occidente de México y, además, plantearnos hipótesis por contrastar con otro tipo de estudios arqueológicos.

En prospectiva, a continuación planteamos una nueva serie de aplicaciones de este método de análisis imagenológico para diferentes tipos del conocimiento arqueológico:

- El diseño de un sistema de excavación asistido por TAC que permita en tiempo real llevar a cabo la excavación física de este tipo de urnas o contextos y, al mismo tiempo, en el modelo computacional, etiquetar cada uno de los elementos, con lo que se tendrá un registro de alta calidad en tres dimensiones. 
- El diseño del sistema de excavación virtual asistida por TAC como herramienta educativa en la formación de estudiantes de arqueología y antropología física, con el objeto de que cuenten con un entrenamiento controlado previo a sus prácticas de campo y, de esta manera, con una experiencia que favorezca el conocimiento técnico y mayor control de los errores humanos dentro de la excavación, esto es, tener el menor daño del contexto arqueológico debido a la inexperiencia.

- En el campo de la divulgación científica, el poder apreciar y manipular los modelos, y visualizar las características y formas de deposición de los elementos de estas urnas funerarias a través de interactivos multimedia y de realidad virtual, será una manera didáctica de aproximarse a estos procesos que sucedieron hace 1000 años y poder presentarlos a manera de hipertextos para los usuarios en los museos o exposiciones.

Cabe señalar que esta técnica arqueológica no invasiva tiene la potencialidad de convertirse en un modelo piloto para la conservación de este tipo de contextos funerarios e incluso de otros más. La adquisición y construcción de un modelo tridimensional de estos objetos y de la totalidad de los elementos que lo componen al interior da la oportunidad de desarrollar investigaciones precisas de estas urnas sin la necesidad de excavarlas, de modo que podemos conservarlas intactas y estabilizarlas tanto para su exposición como para reservarlas en nuestros laboratorios para futuros estudios con nuevas y mejores tecnologías.

A manera de cierre, planteamos que el uso de técnicas imagenológicas para la microexcavación dirigida de contextos arqueológicos de alta complejidad, como son los contextos funerarios, debería ser un requerimiento, en aras de la correcta recuperación de datos y de la preservación en reserva de este tipo de contextos.

\section{Agradecimientos}

Queremos agradecer a las instituciones que han estado involucradas en el proyecto: Centro INAH-Michoacán, Hospital General de México "Dr. Eduardo Liceaga" y Centro de Ciencias Aplicadas y Desarrollo Tecnológico de la Universidad Nacional Autónoma de México (Ccadet-UNAM), por el apoyo para este estudio, así como a la Comisión Nacional del Agua (Conagua) por las facilidades brindadas. Este trabajo no hubiera sido posible sin la colaboración de los arqueólogos Diego Rangel y Érika Ibarra, así como de los estudiantes de la Universidad Autónoma de San Luis Potosí (UASLP), México, Alan García, Elda Margarita Hernández, Gabriela Martínez y Jannet Issaura Peláez.

\section{Referencias}

Acosta, Jorge

1939 “Exploraciones arqueológicas realizadas en el estado de Michoacán durante los años 1937 y 1938", Revista Mexicana de Estudios Antropológicos, tomo III, 2:85-96.

Alcalá, Jerónimo de

2008 Relación de Michoacán, Zamora, El Colegio de Michoacán (Colmich).

Arnauld, Charlotte, Patricia Carot y Marie-France Fauvert-Berthelot

1993 Arqueología de las Lomas en la Cuenca Lacustre de Zacapu, Michoacán, México, México, Centro de Estudios Mexicanos y Centroamericanos (CEMCA), Cuadernos de Estudios Michoacanos, 5.

Bærentzen, J. Andreas, Jens Gravesen, Francois Anton y Henrik Aanæs

2012 "Polygonal Meshes", en Guide to Computational Geometry Processing: Foundations, Algorithms, and Methods, Nueva York, Springer Science \& Business Media, 83-99.

Barker, Philip

1977 Techniques of Archaeological Excavation, Nueva York, Universe Book.

Beta Analytic

s.f. AMS Dating Bones, Antler, and Teeth, documento electrónico disponible en [http://www.radiocarbon.com/carbon-da ting-bones.htm], consultado el 11 de mayo de 2017.

Beucher, Serge

1992 "The Watershed Transformation Applied to Image Segmentation", Scanning Microscopy International, suplemento, 6:299-314.

Binford, L. R.

1972 "Mortuary Practice: Their Study and Their Potential", en L. R. Binford (ed.), An Archaeological Perspective, Nueva York/Londres, Seminar Press, 208-243.

Bonfil Olivera, Alicia

2004 "La muerte entre los otopames arqueológicos del norte del estado de México. Un breve análisis de sus costumbres funerarias", Estudios Mesoamericanos, 6:30-37.

Carot, Patricia y Alberto Susini

1989 "Una práctica funeraria insólita en el occidente: la cremación y pulverización de osamentas humanas", Trace, 16:112-115.

Castro-Leal, Marcia 1986 Tzintzuntzan: capital de los tarascos, Morelia, Gobierno del Estado de Michoacán.

Cerezo-Román, Jessica I. y Howard Williams 2014 "Future Directions for the Archaeology of Cremation", en I. Kuijt, C. Quinn y G. Cooney (eds.), Transformation by Fire: The Archaeology of Cremation in Cultural Context, Tucson, Amerind Foundation/University of Arizona Press, 240-256.

Duncan, William, Andrew Balkansky, Kimberly

Crawford, Heather Lapham y Nathan Meissner 2008 "Human Cremation in Mexico 3,000 Years Ago", Proceedings of the National Academy of Science of the United States of America, 150 (14):5315-5320.

Dunn, J. C.

1973 "A Fuzzy Relative of the ISODATA Process and Its Use in Detecting Compact Well-Separated Clusters", Journal of Cybernetics, 3 (3):32-57. 
Errickson, David y Tim Thompson (eds.)

2017 Human Remains: Another Dimension. The Application of Imaging to the Study of Human Remain, Londres, Academic Press Elsevier.

García Payón, José

1941 "Manera de disponer a los muertos entre los matlatzincas del Valle de Toluca", Revista Mexicana de Estudios Antropológicos, 5:64-78.

Grosman, L.

2016 "Reaching the Point of No Return: The Computational Revolution in Archaeology", Annual Review of Anthropology, 45 (1):129-145, documento electrónico disponible en [http://doi.org/10.1146/annurev-anthro-102215-095946], consultado el 15 de junio de 2016.

Grosman, L., A. Karasik, O. Harush y U. Smilansky

2014 "Archaeology in Three Dimensions-computer-based Methods in Archaeological Research", Journal of Eastern Mediterranean Archaeology Heritage Studies (JEMAHS), 2:48-64.

Grosman, L., O. Smikt y U. Smilansky 2008 "On the Application of 3-D Scanning Technology for the Documentation and Typology of Lithic Artifacts", Journal of Archaeological Science, 35:3101-3110.

Gupta, B., N. Revagade y J. Hilborn

2007 "Poly(lactic acid) fiber: An overview", Progress in Polymer Science, 32 (4):455-482.

Hoffman, E. J., S. C. Huang y M. E. Phelps

1979 "Quantitation in positron emission computed tomography: 1. Effect of object size", Journal of Computer Assisted Tomography, 3 (3):299-308.

Hosler, Dorothy 1994 The Sounds and Color of Power. The Sacred Metallurgical Technology of Ancient West Mexico, Cambridge, MIT Press.

Joukowsky, Martha

1980 A Complete Manual of Field Archaeology Tools and Techniques of Field Work for Archaeologists, Nueva Jersey, Prentice-Hall.

Lorensen, W. E. 1987 "3D Surface Construction Algorithm", Computer Graphics, 21(4):163-169.

Lorensen, W. E. y Harvey E. Cline

1987 "Marching Cubes: A High Resolution 3D Surface Construction Algorithm", ACM Computer Graphics, 21 (4):163-169.

Malik, J., S. Belongie, T. Leung y J. Shi

2001 "Contour and Texture Analysis for Image Segmentation", International Journal of Computer Vision, 43 (1):7-27.

Martínez, Júpiter y Patricia Hernández

2016 "La gente de la sierra alta de Sonora a través de la tomografía axial computada", ponencia presentada en el I Coloquio de Arqueología en Michoacán: Costumbres Funerarias de Michoacán y Áreas Vecinas, Morelia, Michoacán, 2-4 noviembre de 2016, Centro INAH-Michoacán.

McKinley, J. I.

1993 "Cremated Bone", en J. Timby (ed.), Sancton I AngloSaxon Cemetery Excavations Carried out Between 1976 and 1980, Archaeological Journal, 150:243-365.

1997 "The Cremated Human Bone from Burial and Crema-
tion-Related Contexts", en A. P. Fitzpatrick (ed.), Archaeological Excavations on the Route of the A27 Westhampnett Bypass, West Sussex, 1992, vol. 2, Salisbury, Wessex Archaeology Report, 12:55-73.

2013 "Cremation: Excavation, Analysis, and Interpretation of Material from Cremation-Related Contexts", en Liv Nilsson Stutz y Sarah Tarlow (eds.), The Oxford Handbook of the Archaeology of Death and Burial, Oxford, Oxford University Press, 147-171.

Otsu, Nobuyuki

1979 "A Threshold Selection Method from Gray-level Histograms", IEEE Transactions on Systems, Man, and Cybernetics, $9(1): 62-66$.

Peñaloza Meza, Abigaíl

2015 Afinidades biológicas y contextos culturales en los antiguos teotihuacanos, México, Universidad Nacional Autónoma de México-Instituto de Investigaciones Antropológicas (UNAM-IIA).

Pereira, Gregory

1997 "Costumbres funerarias y sociedad del Clásico Tardío en la cuenca de Zacapu, Michoacán", Arqueología, 18:61-84. 2017 "Bioarqueología de las prácticas funerarias", Arqueología Mexicana, 143:50-55.

En prensa "Espacios funerarios en el Michoacán prehispáni$\mathrm{co}^{\prime \prime}$, en F. Agapi (ed.), Michoacán, época prehispánica, Zamora, El Colegio de Michoacán (Colmich).

Piña Chan, Román y Kuniakí Oi

1982 Exploraciones arqueológicas en Tingambato, Michoacán, México, Instituto Nacional de Antropología e Historia (INAH).

Prado, B., A. Gastélum Strozzi, E. Huerta, C. Duwig, O. Zamora, P. Delmas, D. Casasola y J. Márquez

2016 "2, 4-D Mobility in Clay Soils: Impact of Macrofauna Abundance on Soil Porosity", Geoderma, 279:87-96.

Punzo, José Luis

En prensa Una propuesta cronológica prehispánica para la región de Huetamo, en el sureste de Michoacán, México, Arqueología Iberoamericana.

Punzo, José Luis, Diego Rangel, Érika Ibarra y Jesús Zarco 2015 "Primeros datos sobre el uso de adobe y cal en la época prehispánica en la región michoacana del río Balsas medio, México", en Tierra, sociedad, comunidad. $15^{\circ}$ Seminario Iberoamericano de Arquitectura y Construcción con Tierra (SIACOT), Cuenca, SIACOT/Universidad de Cuenca.

2016 "Informe del salvamento arqueológico en el emplazamiento de la presa Chigüero, en el municipio de Huetamo, Michoacán", mecanoescrito, México, Archivo Técnico de la Coordinación Nacional de Arqueología-Instituto Nacional de Antropología (INAH).

Punzo Díaz, José Luis, Alfonso Gastélum Strozzi e Ingris Peláez Ballestas

2016 "Estudio arqueológico no invasivo mediante la reconstrucción de urnas cinerarias de la tierra caliente michoacana", Coordinación Nacional de Arqueología (INAH), 27 de junio, documento electrónico disponible en [http://arqueologia.inah. gob.mx/?p=1263], consultado el 9 de abril de 2017 . 
Reindel, Markus y Günther A. Wagner

2009 "New Methods and Technologies of Natural Sciences for Archaeological Investigations in Nasca and Palpa, Perú", en Markus Reindel y Günther A. Wagner (eds.), New Technologies for Archaeology. Multidisciplinary Investigations in Palpa and Nasca, Perú, Berlin/Heidelberg, Springer-Verlag, 1-17.

Quezada, María Noemí

1996 Los matlatzincas. Época prehispánica y época colonial hasta 1650, México, Universidad Nacional Autónoma de México (UNAM).

Ultimaker

2017 Documento electrónico disponible en [https://ultimaker. com/], consultado el 13 de abril 2017.

Villalpando, Elisa

2016 "Cremaciones de Tradición Trincheras", ponencia presentada en el I Coloquio de Arqueología en Michoacán: Costumbres Funerarias de Michoacán y Áreas Vecinas, Morelia, Michoacán, 2-4 noviembre de 2016, Centro INAH-Michoacán.

\section{Síntesis curricular del/los autor/es}

\section{José Luis Punzo Díaz}

Centro INAH Michoacán,

Instituto Nacional de Antropología e Historia (INAH), México

jose_punzo@inah.gob.mx

Arqueólogo (Escuela Nacional de Antropología e Historia [ENAH], México) maestro en ciencias y humanidades (Universidad Juárez, Durango, México), doctor en arqueología (ENAH). Miembro del Sistema Nacional de Investigadores (SNI) del Consejo Nacional de Ciencia y Tecnología (Conacyt). Ha sido director tanto del Museo de las Culturas del Norte en Paquimé, Chihuahua, como de diversos proyectos arqueológicos en los estados de Durango y Michoacán. Ha fungido como responsable de las zonas arqueológicas de La Ferrería en Durango, así como de Tingambato y Tzintzuntzan en Michoacán; todas en México.

\section{Alfonso Gastélum-Strozzi}

Centro de Ciencias Aplicadas y Desarrollo Tecnológico (Ccadet), Universidad Nacional Autonoma de México (UNAM), México alfonso.gastelum@ccadet.unam.mx

Licenciado en física (Universidad de Guadalajara [UDG], México), maestro en física médica (Universidad Nacional Autónoma de México [unAm], México) y doctor en ciencias de la computación (University of Auckland [UoA], Nueva Zelanda). Desde 2013 trabaja en el Centro de Ciencias Aplicadas y DesarroIlo Tecnológico (Ccadet), anexado al laboratorio de la Unidad de Investigación y Desarrollo Tecnológico (UIDT), fundado en el Hospital General de México "Dr. Eduardo Liceaga" (HGMEL, México). Desde 2014 es miembro del proyecto europeo Protinus, integrado por un equipo multidisciplinario con laboratorios en seis países.

\section{Ingris Peláez Ballestas}

Servicio de Reumatología,

Hospital General de México “Dr. Eduardo Liceaga” (HGM),

Secretaría de Salud, México

ingris.pelaez@salud.gob.mx

Médico cirujano (Escuela de Medicina, Universidad Anáhuac [UA], México), pasante en arqueología (Escuela Nacional de Antropología e Historia [ENAH], México), maestra en ciencias de la salud (Universidad Nacional Autónoma de México [UNAM], México) y doctora en antropología (Instituto de Investigaciones Antropológicas [IIA-UNAM], México). Desde 2012 es profesora de asignatura del posgrado (antropología en salud) de la Facultad de Medicina [UNAM], México). Investigadora en ciencias médicas por la Coordinación Nacional de Institutos y Hospitales de Alta Especialidad de la Secretaría de Salud; miembro del Sistema Nacional de Investigadores (SNI) nivel 2 del Consejo Nacional de Ciencia y Tecnología (Conacyt).

\section{Jesús Zarco Navarro}

Centro INAH-Michoacán,

Instituto Nacional de Antropología e Historia (INAH), México jesuszarconavarro@hotmail.com

Arqueólogo (Escuela Nacional de Antropología e Historia [ENAH], México). Desde el año 2010 ha colaborado en diversos proyectos de investigación arqueológica del Centro INAH-Baja California y del Museo Nacional de Antropología (MNA), enfocados en el estudio de los grupos humanos prehistóricos y misionales que habitaron en la península. En el año 2015 se integró al Centro INAH-Michoacán para realizar diversas actividades de investigación en la región del río Balsas medio y en la Tierra Caliente michoacana, donde ha orientado su trabajo al estudio de las tradiciones tecnológicas líticas desarrolladas ahí por sus antiguos pobladores.

Postulado/Submitted: 12.09.2016

Aceptado/Accepted: 25.04.2017

Publicado/Published: 15.07.2017 